\title{
Desenvolvimento de Crianças Nascidas Pré-Termo Avaliado pelo Teste de Denver-II: Revisão da Produção Científica Brasileira
}

\author{
Development of Children Born Preterm Evaluated by the Denver-II Test: \\ A Review of the Brazilian Scientific Production
}

\author{
Zaira Aparecida de Oliveira Custódio*, Maria Aparecida Crepaldi \& Roberto Moraes Cruz \\ Universidade Federal de Santa Catarina, Florianópolis, Brasil
}

\begin{abstract}
Resumo
Este artigo objetivou analisar a produção científica sobre o desenvolvimento de crianças brasileiras nascidas pré-termo e de muito baixo peso avaliado por meio do Teste de Denver-II, no período de 2000 a 2009. Realizou-se levantamento bibliográfico de estudos empíricos indexados nas bases de dados Medline, Lilacs e Scielo, por meio da combinação das palavras chave: pré-termo, muito baixo peso, fatores de risco, desenvolvimento, Denver-II. Foram identificados 26 artigos e selecionados oito conforme critérios de inclusão. A maioria dos estudos avaliou crianças pré-termo entre 5 a 24 meses de idade. Os estudos identificaram associação entre as variáveis neonatais e ambientais e o desempenho no Teste de Denver-II. Crianças nascidas pré-termo devem ser acompanhadas ambulatorialmente para prevenir e detectar riscos no desenvolvimento.

Palavras-chave: Pré-termo, muito baixo peso, fatores de risco, desenvolvimento, Denver-II.

Abstract

This article aims to analyze the scientific literature on the development of children born preterm with very low birth weight assessed by the Denver-II test in the period from 2000 to 2009. A survey of empirical literature indexed in Medline, Lilacs and Scielo was carried out through the combination of the keywords: preterm, very low birth weight, risk factors, development, and Denver-II. Twenty six articles were identified and eight were selected following the inclusion criteria. Most studies evaluated preterm children between 5 and 24 months of age. The studies identified a significant association between neonatal and environmental variables and the performance on Denver-II Test. Children born preterm must be followedup at outpatient Units to prevent and detect risks in development.

Keywords: Preterm, very low birth weight, risk factors, development, Denver-II.
\end{abstract}

O nascimento pré-termo $(<37$ semanas de idade gestacional) e a condição de muito baixo peso $(<1500 \mathrm{~g})$ e de extremo baixo peso $(<1000 \mathrm{~g})$ estão entre as causas importantes de mortalidade infantil no Brasil, segundo Segre (2002). No final da década de 90, a Rede Brasileira de Pesquisas Neonatais mostrava que a expectativa de sobrevida para crianças nascidas pré-termo de 750-1000g era de 66 a $73 \%$ e para crianças nascidas de 500-749g variava de 9 a 44\% (Leone et al., 2001). A morbidade neonatal aumenta na medida em que diminui a idade gestacional da criança e o peso ao nascer. Além disso, o grau de educação materna e o nível socioeconômico da família contribuem para o desenvolvimento do recém-nascido pré-termo (Penalva \& Schwartzman, 2006).

\footnotetext{
*Endereço para correspondência: Hospital Universitário Polydoro Ernani de São Thiago, Universidade Federal de Santa Catarina, Rua Prof a Maria Flora Pausewang, s/n, Bairro Trindade, Caixa Postal 5199, Florianópolis, SC, Brasil 88040-900. E-mail: zaira@hu.ufsc.br
}

Embora tenha havido avanços científicos e tecnológicos nas duas últimas décadas associados a importantes mudanças na assistência obstétrica e neonatal, permitindo a sobrevivência de recém-nascidos pré-termo de extremo baixo peso, as seqüelas da prematuridade podem ser graves e penosas, tanto para o bebê quanto para a sua família (Rugolo, 2005). Estudos de Fraga, Linhares, Carvalho e Martinez (2008) e Laucht, Esser e Schmidt (1997) têm demonstrado a grande vulnerabilidade em que se encontram as crianças nascidas pré-termo, influenciando assim, seu desenvolvimento e integração no ambiente. A condição de prematuridade aumenta na criança a ocorrência de problemas motores (Halpern, Giugliani, Victora, Barros, \& Horta, 2000), cognitivos (Linhares, Chimello, Bordin, Carvalho, \& Martinez, 2005), de linguagem (Carvalho, Linhares, \& Martinez, 2001) e de comportamento (Carvalho et al., 2001; Linhares, Carvalho, Bordin, \& Jorge, 1999).

Com base nos autores supracitados, o nascimento prétermo se constitui em fator de risco, em si mesmo, para o 
desenvolvimento da criança. A definição de fatores de risco inclui a interação dinâmica de uma série de condições biológicas e ambientais que impedem o pleno desenvolvimento da criança (Laucht et al., 1997; Laucht, Esser, \& Schmidt, 2001; Resegue, Puccini, \& Silva, 2007). Os riscos ou adversidades não costumam estar isolados ou independentes, segundo Sapienza e Pedromônico (2005), pois fazem parte do contexto social, envolvendo fatores políticos, socioeconômicos, ambientais, culturais, familiares e genéticos. Isto aparece, por exemplo, em um estudo longitudinal realizado por Laucht et al. (2001), em que os autores acompanharam o comportamento de crianças nascidas com baixo peso (risco biológico) e problemas familiares (risco psicossocial). Os resultados sugerem que quando há riscos conjugados, o efeito simultâneo é amplamente aditivo.

Nesse sentido, Halpern e Figueiras (2004) orientam para a importância de se avaliar os fatores de risco psicossociais e biológicos associados, e seu impacto no desenvolvimento da criança. Advertem para a importância da prevenção e da intervenção precoce, além do acompanhamento das crianças em situação de risco. Dessa forma, para Resegue, Puccini e Silva $(2007,2008)$ a triagem de risco deve ser precoce, já a partir do período neonatal com base no conhecimento dos fatores de riscos associados a alterações do desenvolvimento. Essas alterações podem ser confirmadas ou não por meio de acompanhamento sistemático e de avaliação diagnóstica (Halpern, \& Figueiras, 2004; Linhares et al., 2004; Resegue et al., 2008).

Em um estudo retrospectivo, Resegue et al. (2008) analisaram a associação entre fatores de risco para o atraso de desenvolvimento e as alterações observadas em crianças nascidas pré-termo acompanhadas ambulatorialmente. O referido estudo aponta para a importância que deve ser dada ao desenvolvimento das crianças nascidas com baixo peso e com histórias de intercorrências neonatais, servindo estes de marcadores importantes para definir o acompanhamento do desenvolvimento dessas crianças.

Linhares et al. (2004), ressaltam a importância da intervenção preventiva como uma estratégia de proteção ao desenvolvimento da criança que nasceu prematura. Os programas de intervenção, segundo Linhares et al. (2000), devem considerar pelo menos o período dos três primeiros anos de vida, que inclui a aquisição de habilidades psicomotoras e de linguagem e o período de seis a sete anos, quando a criança inicia o desafio do ensino fundamental.

Na perspectiva de detectar os riscos para o desenvolvimento neuropsicomotor da criança e realizar diagnósticos para o planejamento de intervenções precoces, os profissionais de saúde se utilizam de instrumentos de medida. Porém, segundo Santos, Araújo e Porto (2008), no Brasil, os serviços que realizam o seguimento de crianças nascidas pré-termo adotam instrumentos de avaliação de forma assistemática, tais como as escalas de desenvolvimento. Conforme esses autores, há uma escassez de instrumentos de avaliação para a identificação precoce, padronizados para a população brasileira.

Santos et al. (2008) identificaram os instrumentos mais utilizados em pesquisas nacionais e internacionais na avaliação para triagem e identificação precoce de atraso no desenvolvimento em crianças nascidas pré-termo. Os instrumentos identificados foram: a Escala Motora de Alberta (Alberta Infant Motor Scale - AIMS), a Avaliação dos Movimentos da Criança (Movement Assessment of Infant - MAI), o Teste Infantil de Desempenho Motor (Test of Infant Motor Performance - TIMP), a Avaliação dos Movimentos Generalizados (General Movements - GM). Além destes, o Teste de Triagem de Desenvolvimento de Denver-II foi considerado o mais utilizado.

O Teste de Denver-II, como é conhecido atualmente, teve sua versão original desenvolvida por Frankenburg e Dodds em 1967 na cidade do Colorado, EUA, e era chamado de Teste de Desenvolvimento de Denver (TDD). O nome "Denver" se deve ao fato de que este teste foi criado na Universidade de Colorado Medical Center, em Denver. As dificuldades apresentadas pelo TDD obstaculizaram sua aplicação em pesquisas, o que levou a uma nova normatização com a revisão de alguns itens e atualização das normas por idade, resultando, assim, no Teste de Triagem de Desenvolvimento de Denver-II / Denver Developmental Screening Test - DDST-II (Frankenburg, Dodds, Archer, Shapiro, \& Bresnik, 1992), traduzido e adaptado no Brasil por Figueiras, Pedromônico, Sales e Figueiras (2000).

Assim como o TDD, o Teste de Denver-II é um instrumento de triagem de fácil e rápida aplicação para detectar atrasos no desenvolvimento em crianças de 0 a 6 anos de idade, bem como de treinamento fácil, utilizado por profissionais da área da saúde em geral, segundo Halpern et al. (2000). É composto de 125 itens divididos em quatro áreas, a saber: pessoal-social (25 itens) - envolve aspectos da socialização da criança dentro e fora do ambiente familiar; motricidade fina (29 itens) - coordenação olho-mão, manipulação de pequenos objetos; linguagem (39 itens) - produção de som, capacidade de reconhecer, entender e usar a linguagem; motricidade ampla (32 itens) - controle motor corporal, sentar, caminhar, pular e os demais movimentos realizados pela musculatura ampla (Halpern et al., 2000).

Os itens são administrados diretamente à criança, sendo que em alguns deles é solicitado à mãe ou cuidador que informe se a criança realiza ou não determinada tarefa (Halpern et al., 2000). Na folha de aplicação faz-se uma linha vertical sobre a idade da criança e esta passa pelas quatro áreas do desenvolvimento que serão avaliadas, mostrando os itens que deverão ser aplicados para a idade referida. A linha vertical corta os retângulos existentes em cada item e estes são divididos, sendo uma parte branca ( 25 a $75 \%$ não executam ainda a tarefa) e outra sombreada (75 a $90 \%$ já deveriam executar tais tarefas).

Estudos que abordam a triagem de risco para desenvolvimento de crianças nascidas pré-termo e de muito 
baixo peso, no Brasil, são de fundamental importância considerando-se a necessidade de dados sobre a detecção precoce de problemas de desenvolvimento, para o planejamento de intervenções que promovam o desenvolvimento. O objetivo deste estudo é realizar uma revisão da literatura, sobre o desenvolvimento de crianças brasileiras nascidas pré-termo e de muito baixo peso, avaliado por meio do Teste de Denver-II, no período de 2000 a 2009. O presente trabalho justifica-se tendo em vista que é necessário que se tenha acesso no Brasil, a instrumentos de avaliação de bebês nascidos pré-termo, que sejam simples e de fácil aplicação, mas fidedignos. Esta medida é importante para atender ao Programa de vigilância do crescimento e desenvolvimento infantil (Ministério da Saúde, 2002) desenvolvido na atenção básica, que preconiza a importância da avaliação e intervenção preventiva a fim de detectar e intervir em potencial de agravos para o desenvolvimento de crianças. Outro aspecto a considerar é que se trata de uma revisão original tendo em vista que nenhum trabalho desta natureza foi encontrado na literatura brasileira sobre o tema.

\section{Método}

Realizou-se revisão bibliográfica nas bases de dados informatizadas Medline, Lilacs e Scielo, por meio da combinação em conjunto dos seguintes descritores: pré-termo, muito baixo peso, fatores de risco, desenvolvimento, Denver-II e, seus correspondentes em inglês: preterm, very low birth weight, risk factors, development, DenverII. Foram definidos como critérios de inclusão artigos empíricos de língua portuguesa e inglesa, desenvolvidos no Brasil com crianças nascidas pré-termo e de muito baixo peso, abrangendo as fases do nascimento e primeiro ano de vida, pré-escolar (dois a cinco anos) ou o início da fase escolar (seis anos), publicados nos anos de 2000 a 2009, em periódicos ou revistas especializadas e indexadas nas referidas bases de dados. Foram identificados 26 artigos, dos quais selecionou-se 8, de acordo com os critérios de inclusão, que foram, por sua vez, localizados no sistema on line.

\section{Resultados}

A Tabela 1 apresenta os resultados referentes ao tamanho da amostra e idade das crianças estudadas.

Na Tabela 1 verifica-se que o tamanho da amostra dos estudos variou de 20 a 178 crianças nascidas pré-termo com baixo peso e muito baixo peso de ambos os sexos. Dois estudos compararam grupos de crianças nascidas pré-termo de diferentes faixas etárias e um estudo agrupou os participantes pré-termo em grupo com risco de atraso de desenvolvimento e grupo com desenvolvimento normal. Com relação à idade, em 5 estudos as crianças estavam entre 5 e 14 meses, dois estudos avaliaram crianças que se encontravam com 12 e 24 meses e em um estudo as crianças estavam na fase pré-escolar (4-5 anos).
Na Tabela 2 encontram-se os dados relativos aos tipos de estudos, métodos, técnicas utilizadas e análises de dados.

Tabela 1

Tamanho da Amostra e Idade das Crianças

\begin{tabular}{ll}
\hline Variável & $N$ \\
\hline Tamanho da amostra & 2 \\
20 & 1 \\
30 & 1 \\
32 & 1 \\
80 & 1 \\
69 & 1 \\
87 & 8 \\
178 & 5 \\
\hline Total & 2 \\
\hline Idade das crianças em meses & 1 \\
5 e 14 & 8 \\
42 e 24 & e 60 \\
\hline Total & \\
\hline
\end{tabular}

Tabela 2

Tipos de Estudos, Métodos, Técnicas Utilizadas e Análises de Dados

\begin{tabular}{ll}
\hline Variável & $N$ \\
\hline Tipo de Estudo & \\
Longitudinal & 5 \\
Transversal & 3 \\
\hline Total & 8 \\
\hline
\end{tabular}

Método

Levantamento de dados 7

Levantamento de dados e Observação

Total 8

Técnica Utilizada

Escalas/Exame Neurológico 3

Entrevista/Prontuário 1

Escalas/Entrevista/Prontuário 1

Observação 1

Questionário 1

Entrevista 1

Total 8

Análise de Dados

Quantitativa 8

$\begin{array}{ll}\text { Total } & 8\end{array}$ 
Observou-se que em cinco estudos as crianças estavam sendo acompanhadas em ambulatório de seguimento durante a coleta de dados, em um estudo as crianças foram acompanhadas no referido ambulatório nos seus dois primeiros anos de vida e em dois outros estudos este tipo de acompanhamento não foi referido. Observa-se na Tabela 2 que a maioria dos trabalhos seguiu o delineamento do tipo Longitudinal e o método predominante foi o Levantamento de dados. Os trabalhos, além de analisarem os aspectos do desenvolvimento da criança por meio do Teste de Denver-II, utilizaram-se de outras técnicas a fim de avaliar variáveis da própria criança, do seu cuidador e do ambiente. Três estudos utilizaram roteiro de entrevista ou questionário, dois estudos fizeram uso do prontuário do paciente e, em um estudo utilizou-se a observação sistemática do comportamento. Verificou-se a combinação de escalas e exames neurológicos em outros três estudos. Todos os trabalhos foram analisados quantitativamente.

Os trabalhos avaliaram o risco no desenvolvimento nas áreas pessoal-social, linguagem, motricidade fina e ampla, por meio do Teste de Denver-II. Contudo, também relacionaram, de modo geral, as seguintes variáveis da criança: sexo, peso, idade gestacional, índice de Apgar de $1^{\circ}$ e $5^{\circ}$ minutos, índice de risco clínico neonatal, tempo de internação, duração da amamentação, desenvolvimento cognitivo e comportamental, direção do olhar, sorriso, vocalização, manipulação, locomoção (variáveis do comportamento exploratório em situação de brincar).

Um estudo investigou as variáveis maternas relacionadas ao desenvolvimento da criança, como: idade, estado marital, trabalho externo, pré-natal, intercorrências na gestação, tipo de parto; as variáveis maternas ansiedade, depressão e disforia, foram relacionadas em 1 estudo; o número de filhos, apoio a gestação e se fumou na gestação foram variáveis presentes em outro estudo; as variáveis do ambiente familiar (ocupação dos pais, situação conjugal dos pais, número de cômodos, números de pessoas que moram na casa e tipo de moradia) relacionaram-se em outro trabalho. A variável escolaridade dos pais foi investigada em três estudos e a variável renda esteve presente em dois estudos.

A partir da leitura e análise dos artigos componentes da amostra os resultados obtidos foram assim agrupados: prevalência de atraso no desenvolvimento da criança; associação de indicadores de risco para atraso de desenvolvimento e outras variáveis neonatais e ambientais; atraso no desenvolvimento e a relação com alterações no desenvolvimento avaliadas por outros instrumentos. Segue, abaixo, a apresentação de cada um desses grupos.

Dos estudos analisados, três avaliaram a prevalência de atraso no desenvolvimento da criança (Chermont, Cunha, Sales, Moraes, \& Malveira, 2005; Espírito Santo, Portuguez, \& Nunes, 2009; Halpern et al., 2000). A prevalência de suspeita de atraso no desenvolvimento neuropsicomotor em crianças, aos 12 meses de vida e seus possíveis determinantes foi verificada no estudo de
Halpern et al. (2000). Da amostra de 1.363 crianças, 178 eram crianças nascidas pré-termo com baixo ou muito baixo peso. As crianças que nasceram com menos de $2.000 \mathrm{~g}$ tiveram um risco de apresentar um teste suspeito no Denver-II, quatro vezes maior, quando comparadas com as mais pesadas. As crianças pré-termo, por sua vez, apresentaram uma chance $60 \%$ maior de teste suspeito de atraso no desenvolvimento neuropsicomotor.

O Teste de Denver-II foi utilizado no estudo Chermont et al. (2005) para avaliar o desenvolvimento de crianças nos primeiros 2 anos de vida, atendidas num programa de seguimento de recém-nascidos pré-termo e de baixo peso de um hospital universitário. Os resultados do referido estudo indicaram que a maioria das crianças manifestou alteração em pelo menos uma das quatro áreas estudadas.

Já, Espírito Santo et al. (2009) avaliaram o desenvolvimento cognitivo e comportamental em idade pré-escolar (4-5 anos) de crianças nascidas pré-termo e com baixo peso. Os achados sugerem que recém-nascidos pré-termo com baixo peso, mesmo com o peso predominantemente entre 1.500 e 2.500 gramas, possuem prevalência relativamente alta de transtornos cognitivos e comportamentais em idade pré-escolar em comparação com os controles descritos na literatura. Os resultados considerados como atraso no Teste de Denver-II e nos demais instrumentos foram associados com QI baixo.

Três estudos avaliaram a associação de indicadores de risco para atraso de desenvolvimento e outras variáveis neonatais e ambientais (Fraga et al., 2008; Kreling, Brito, \& Matsuo, 2006; Nobre, Carvalho, Martinez, \& Linhares, 2009). Os fatores de risco para o desenvolvimento neuropsicomotor de recém-nascidos de muito baixo peso, aos 12 meses de idade corrigida foram avaliados no estudo de Kreling et al. (2006), os quais observaram que $37,9 \%$ dos bebês com peso inferior a $1.000 \mathrm{~g}$ apresentaram resposta alterada no Teste de Denver-II. Nesse estudo, as variáveis maternas gestacionais não mostraram correlação com o desenvolvimento, como também os indicadores socioeconômicos de renda, emprego e a escolaridade da mãe.

Nessa direção, Fraga et al. (2008) realizaram estudo longitudinal a fim de comparar um grupo com bebês e suas mães que apresentaram indicadores de ansiedade, depressão e/ou disforia durante o período neonatal, com um grupo formado por bebês e suas mães, sem indicadores emocionais. O Teste de Denver-II foi aplicado nas crianças de ambos os grupos, as quais se encontravam com 5-7 e 10-11 meses de idade corrigida e, apresentaram indicadores de desenvolvimento típico no primeiro ano de vida, independentemente da presença de ansiedade e depressão materna.

Por outro lado, um estudo de Nobre et al. (2009) mostrou que o risco relativo para problemas de desenvolvimento de crianças nascidas pré-termo nas faixas de 5-7 e 10-14 meses de idade corrigida foi, respectivamente, de 
$20 \%$ e $27 \%$. Tais indicadores foram relacionados com variáveis neonatais e do ambiente familiar. Na comparação do desempenho dos bebês, avaliados nas duas faixas etárias foi observado uma diferença significativa apenas na área da Linguagem. Segundo os autores, as crianças nascidas com menor peso e idade gestacional, que permaneceram mais tempo hospitalizadas e com situações familiares adversas apresentaram mais problemas de desenvolvimento.

Finalmente, dois estudos relacionaram atraso no desenvolvimento, detectado pelo Teste de Denver-II, com outras alterações no desenvolvimento da criança, avaliadas por outros instrumentos (Lopes, Martinez, \& Linhares, 2008; Schirmer, Portuguez, \& Nunes, 2006). A influência da idade gestacional e o peso ao nascimento na aquisição da linguagem e, neurodesenvolvimento em crianças de 3 anos de idade nascidas pré-termo foi avaliada por Schirmer et al. (2006), os quais demonstraram que a idade gestacional está associada com alteração comportamental e atraso na aquisição da linguagem. Nesse estudo, o resultado do Teste de Denver-II alterado tanto aos 12 meses quanto aos 24 meses, estava associado com o atraso na aquisição de linguagem receptiva e expressiva aos 3 anos de idade, avaliada por meio da Escala Infantil Bayley II.

O estudo de Lopes et al. (2008), por sua vez, dividiu dois grupos de crianças nascidas pré-termo e com baixo peso, aos 10 meses de idade corrigida, de acordo com o desempenho no Teste de Denver-II (normal e risco de atraso no desenvolvimento) a fim de avaliar o comportamento exploratório na situação de brincar. Os autores verificaram uma coerência entre os resultados da observação sistemática do comportamento e da avaliação do desenvolvimento. Ou seja, os bebês com classificação normal no desenvolvimento apresentaram melhor resultado na locomoção, na interação com a observadora, por meio do olhar e na expressão facial.

\section{Discussão}

Os achados demonstraram relação significativa entre o desempenho do desenvolvimento, avaliado pelo Teste de Denver-II e as variáveis neonatais. Especialmente o peso e a idade gestacional foram associados com maior risco das crianças apresentarem resposta alterada no DenverII, o que indica potencial para problemas de desenvolvimento (Chermont, et al., 2005; Halpern et al., 2000; Kreling et al., 2006; Nobre et al., 2009). As crianças com características relacionadas a estas variáveis apresentaram atraso na aquisição da linguagem e baixo desempenho cognitivo e motor (Espírito Santo et al., 2009; Schirmer et al., 2006). Este dado, por si, já é um indicador importante para equipes de saúde que necessitam fazer um acompanhamento de bebês pré-termo após a alta, na atenção primária e secundária em saúde, tendo em vista que o desempenho cognitivo, por exemplo, é um importante preditor de desempenho escolar nos anos posteriores (Linhares et al., 2005).

Com relação ainda as variáveis neonatais (peso e idade gestacional), bebês que apresentaram alto índice de risco neonatal e que permaneceram mais tempo internados na UTI Neonatal apresentaram mais risco no desenvolvimento (Nobre et. al., 2009). Por outro lado, destacou-se o tempo de amamentação, de forma que a chance do Teste de Denver-II apresentar suspeita de atraso é menor, quanto maior for o tempo da criança em aleitamento materno (Halpern et al., 2000). Pode-se concluir que especial atenção deve ser dada a triagem de risco em crianças com grande vulnerabilidade neonatal, a fim de minimizar os problemas de desenvolvimento. Assim como, oferecer estímulo e apoio à amamentação por parte dos profissionais que acompanham a díade mãe-bebê desde a unidade neonatal.

A avaliação das crianças com 1 ano de idade corrigida parece não ser adequada para detectar a influência das variáveis gestacionais maternas, segundo estudo de Kreling et al., (2006), pois não mostraram correlação com o desenvolvimento da criança, assim como a renda, emprego e escolaridade. No entanto, os indicadores socioeconômicos renda familiar e escolaridade dos pais estão fortemente associados com atraso no desenvolvimento aos 12 meses, no estudo de Halpern et al. (2000). Segundo este estudo as crianças que vivem em ambientes empobrecidos apresentaram 50\% mais riscos de terem suspeita de atraso.

O estudo que identificou as variáveis maternas ansiedade e depressão não encontrou associação entre estas e o desenvolvimento no primeiro ano de vida, mostrando que os grupos avaliados foram mais semelhantes do que diferentes em seus indicadores (Fraga et al., 2008). Este estudo foi realizado em um serviço, cujas mães das crianças receberam acompanhamento psicológico para enfrentamento do estresse e ansiedade, devido à internação neonatal da criança. Essa condição pode ter contribuído para criar um contexto de suporte às mães e minimizar os efeitos adversos da ansiedade, contribuindo no desenvolvimento dos bebês.

No que concernem as variáveis ambientais, nos achados de Nobre et al. (2009) a variável número de pessoas que residem com a criança nascida pré-termo mostrou relação com o desenvolvimento da linguagem. Esse estudo indicou que as crianças que residem com maior número de pessoas na casa (em torno de 4 pessoas) apresentaram menor risco na área da linguagem avaliada no Teste de Denver-II. A característica paridade esteve relacionada com a suspeita de atraso no desenvolvimento, conforme estudo de Halpern et al. (2000). As crianças com mais de três irmãos tiveram mais chances de um desempenho no teste com suspeita de atraso. Infere-se que nas famílias com maior número de filhos, havia menos disponibilidade materna para estimular a criança. 
Custódio, Z. A. O., Crepaldi, M. A. \& Cruz, R. M. (2012). Desenvolvimento de Crianças Nascidas Pré-Termo Avaliado pelo Teste de Denver-II: Revisão da Produção Científica Brasileira.

A comparação entre grupos extraídos da amostra de pré-termos, nos quais estavam de um lado bebês com risco de atraso e, de outro, bebês com desenvolvimento típico, mostrou a importância da avaliação do desenvolvimento desses bebês para melhor especificação do risco (Lopes et al., 2008). Os bebês com melhor desempenho no Teste de Denver-II exploraram melhor os brinquedos. Segundo os autores, os achados remetem para a importância da avaliação e intervenção preventiva na população de pré-termos, a fim de amenizar as dificuldades observadas em relação aos comportamentos de exploração do ambiente.

Para Chermont et al. (2005), as crianças nascidas pré-termo devem ser rigorosamente acompanhadas num programa de seguimento por uma equipe multidisciplinar para prevenir e detectar precocemente os riscos no desenvolvimento. Porém, é necessário que a avaliação seja repetida, segundo Halpern et al. (2000), especialmente nos primeiros anos de vida quando o desenvolvimento é mais dinâmico e o impacto dos atrasos mais importantes.

O Teste de Denver-II foi adequado para a avaliação do desenvolvimento de crianças nascidas pré-termo e de muito baixo peso, conforme aponta Kreling et al. (2006). Contudo, como os demais testes de triagem, o Teste de Denver-II alerta para um risco no desenvolvimento que pode ser confirmado ou não, sistematicamente. A avaliação do desenvolvimento diz respeito a um dado momento, o que não determina de forma definitiva um atraso no desenvolvimento da criança (Halpern, \& Figueiras, 2004; Resegue et al., 2008).

Considerando o escasso número de pesquisas identificadas para esta revisão, sobre o desenvolvimento de crianças brasileiras nascidas pré-termo que utilizaram o Teste de Denver-II, julga-se de extrema importância a realização de outros estudos com o objetivo de rastrear o risco para atrasos no desenvolvimento nessa população. A maior parte destes estudos foi realizada em hospitais universitários e/ou serviços especializados de alta complexidade, o que não invalida a utilização do referido teste nos serviços de atenção primária e secundária pela facilidade de sua aplicação e fidedignidade de seus resultados. Porém, seria importante também a realização de novos estudos no contexto específico de atenção primária e secundária à saúde.

Assim, constata-se que os estudos ainda são incipientes no Brasil, feitos com amostras pequenas e com método que se aplica apenas a caracterização destas amostras. Apesar desta evidência, os estudos apresentados nesta revisão apontam o Teste de Denver-II como uma ferramenta que pode ser usada em larga escala para avaliação de crianças pré-termo considerando-se, porém, que esta avaliação seja feita periodicamente, e que o teste esteja associado a outros instrumentos adequados, para cada faixa etária. Esta medida poderá subsidiar Programas de atenção à saúde da criança no Sistema Único de Saúde.

\section{Referências}

Carvalho, A. E. V., Linhares, M. B. M., \& Martinez, F. E. (2001). História de desenvolvimento e comportamento de crianças nascidas pré-termo e baixo peso $(1.500 \mathrm{~g})$. Psicologia: Reflexão Crítica, 14(1), 1-33.

Chermont, A. G., Cunha, M. S., Sales, L. M. M., Moraes, A. N., \& Malveira, S. S. (2005). Avaliação do desenvolvimento pela escala de Denver-II, de recém-nascidos prematuros de baixo peso. Revista Paraense de Medicina, 19(2), 59-66.

Espírito Santo, J. L., Portuguez, M. W., \& Nunes, M. L. (2009). Status cognitivo-comportamental de prematuros de baixo peso ao nascer em idade pré-escolar que vivem em país em desenvolvimento. Jornal de Pediatria, 85(1), 35-41.

Figueiras, A. C. M., Pedromônico, M., Sales, L., \& Figueiras, S. (2000). Manual para vigilância do desenvolvimento da criança de 0 a 2 anos de idade na atenção primária à saúde. Belém, PA: Secretaria Municipal de Saúde de Belém.

Fraga, D. A., Linhares, M. B. M., Carvalho, A. E. V., \& Martinez, F. E. (2008). Desenvolvimento de bebês nascidos pré-termo e indicadores emocionais maternos. Psicologia: Reflexão e Crítica, 21(1), 33-41.

Frankenburg, W. K., Dodds, J., Archer, P., Shapiro H., \& Bresnick, B. (1992). The Denver II: A major revision and restandardization of the Denver Developmental Screening Test. Pediatrics, 89, 91-97.

Halpern, R., \& Figueiras, A. C. M. (2004). Influências ambientais na saúde mental da criança. Jornal de Pediatria, 80(2), 104-110.

Halpern, R., Giugliani, E. R. J., Victora, C. G., Barros, F. C., \& Horta, B. L. (2000). Fatores de risco para suspeita de atraso no desenvolvimento neuropsicomotor aos 12 meses de vida. Jornal de Pediatria, 76(6), 421-428

Kreling, K. C. Á., Brito, Â. S. J., \& Matsuo, T. (2006). Fatores perinatais associados ao desenvolvimento neuropsicomotor de prematuros de muito baixo peso. Pediatra, 28(2), 98-108.

Laucht, M., Esser, G., \& Schmidt, M. H. (1997). Developmental outcome of infants born with biological and psychosocial risks. Journal Child Psychology and Psychiatry, 38(7), 843-853.

Laucht, M., Esser, G., \& Schmidt, M. H. (2001). Differential development of infants at risk for psychopathology: The moderating role of early maternal responsivity. Developmental Medicine \& Child Neurology, 43(5), 292-300.

Leone, C. R., Sadeck, L. S., Vaz, F. A., Almeida, M. F., Draque, C. M., Guinsburg, R., et al. (2001). Brazilian Neonatal Research Netork (BNRN): Very-low birth weight (VLBW) infant morbidity and mortality. Pediatric Research, 49, 405A.

Linhares, M. B. M., Carvalho, A. E. V., Bordin, M. B. M., Chimello, J. T., Martinez, F. E., \& Jorge, S. M. (2000). Prematuridade e muito baixo peso como fatores de risco ao desenvolvimento da criança. Cadernos de Psicologia e Educação, 10, 60-69.

Linhares, M. B. M., Carvalho, A. E. V., Bordin, M. B. M., \& Jorge, S. M. (1999). Suporte psicológico ao desenvolvimento de bebês pré-termo com peso de nascimento $<1500 \mathrm{~g}$ : Na UTI-neonatal e no segmento longitudinal. Temas em Psicologia, 7(3), 245-262. 
Linhares, M. B. M., Carvalho, A. E. V., Padovani, F. H. P., Bordin, M. B. M., Martins, I. M. B., \& Martinez, F. E. (2004). A compreensão do fator de risco da prematuridade sob a ótica desenvolvimental. In E. M. Marturano, M. B. M. Linhares, \& S. R. Loureiro (Eds.), Vulnerabilidade e proteção: Indicadores na trajetória de desenvolvimento do escolar. São Paulo, SP: Casa do Psicólogo.

Linhares, M. B. M., Chimello, J. T., Bordin, M. B. M., Carvalho, A. E. V., \& Martinez, F. E. (2005). Desenvolvimento psicológico na fase escolar de crianças nascidas pré-termo em comparação com crianças nascidas a termo. Psicologia: Reflexão e Crítica, 18(1), 109-117.

Lopes, D. V., Martinez, F. E., \& Linhares, M. B. M. (2008). Comportamento exploratório de bebês nascidos pré-termo em situação de brincar. Psicologia em Estudo, 13(4), 867-874.

Ministério da Saúde. (2002). Saúde da criança: Acompanhamento do crescimento e desenvolvimento infantil. Brasília, DF: Autor.

Nobre, F. D. A., Carvalho, A. E. V., Martinez, F. E., \& Linhares, M. B. M. (2009). Estudo longitudinal do desenvolvimento de crianças nascidas pré-termo no primeiro ano de vida. Revista Interamericana de Psicologia, 43(3), 442-450.

Penalva, O., \& Schwartzman, J. S. (2006). Estudo descritivo do perfil clínico-nutricional e do seguimento ambulatorial de recém-nascidos prematuros atendidos no Programa Método Mãe Canguru. Jornal de Pediatria, 82(1), 33-39.

Resegue, R., Puccini, R. F., \& Silva, E. M. K. (2007). Fatores de risco associados a alterações no desenvolvimento da criança. Pediatria, 29(2), 117-128.

Resegue, R., Puccini, R. F., \& Silva, E. M. K. (2008). Risck factors associated with developmental abnormalities among high-risk children attended at a multidisciplinary clinic. São Paulo Medical Journal, 126(1), 4-10.

Rugolo, L. M. (2005). Crescimento e desenvolvimento a longo prazo do prematuro extremo. Jornal de Pediatria, 81(1), 101-110.

Santos, R. S., Araújo, A. P. Q. C., \& Porto, M. A. S. (2008). Diagnóstico precoce de anormalidades no desenvolvimento em prematuros: Instrumentos de avaliação. Jornal de Pediatria, 84(4), 289-299.

Sapienza, G., \& Pedromônico, M. R. M. (2005). Risco, proteção e resiliência no desenvolvimento da criança e do adolescente. Psicologia em Estudo, 10(2), 209-216.

Schirmer, C. R., Portuguez, M. W., \& Nunes, M. L. (2006). Clinical Assessment of language development in children at age 3 years that were born preterm. Arquivos de Neuro-Psiquiatria, 64(4), 926-931.

Segre, C. A. M. (2002). Perinatologia: Fundamentos e prática. São Paulo, SP: Sarrier. 